\title{
¿CÓMO CIRCULA EL VIENTO EN LOS TRÓPICOS? AVANCES EN LA DISCIPLINA CLIMÁTICA DURANTE LA EDAD MODERNA*
}

\author{
Jorge OlCina CANTOS \\ Universidad de Alicante \\ jorge.olcina@ua.es
}

\section{Resumen}

Los siglos XVII y XVIII son esenciales para comprender la evolución posterior de las ciencias del tiempo y clima. Junto a la mejora en los sistemas y métodos de observación de los elementos climáticos, se formulan una serie de propuestas que intentarán explicar el funcionamiento de la circulación atmosférica y, en particular, de los vientos en las diferentes zonas terrestres. La preocupación principal será la comprensión de los alisios del ámbito intertropical, por su importancia para la navegación marítima. Científicos, geógrafos y filósofos aportan propuestas que irán, progresivamente, mejorando la interpretación de la circulación de los vientos alisios a lo largo de estas dos centurias. El trabajo muestra los ensayos principales que se desarrollan en este período y que serán la base de las mejoras en la ciencia climática de los siglos posteriores.

Palabras clave: ciencia climática, alisios, trópicos, navegación marítima, edad Moderna.

\section{Abstract}

How does blow the wind in the tropics? Climatology in the Modern age

Seventeenth and eighteenth centuries are essential to understanding the subsequent evolution on weather and climate sciences. Beside the improvement in the systems and methods of meteorological phenomena, a serie of proposals that try to explain

* El presente estudio se inscribe en el marco del Proyecto de Investigación «Oscilaciones climáticas y crisis agrarias en el levante español durante la pequeña Edad del Hielo» (HAR2013-44972-P) financiado por el Ministerio de Economía y Competitividad para el trienio 2014-2016, bajo la dirección del Dr. Alberola Romá. 
the performance of atmospheric circulation and, in particular, the motion of winds in different terrestrial zones, are formulated. Main concern will be the understanding of the trade winds in tropics, because of its importance for sea navigation. Scientists, geographers and philosophers contribute proposals that will be progressively get better the interpretation of the circulation of trade winds throughout these two centuries. This work shows the main papers that are developed in this period and that will be the basis of the improvements in the climatic science of the later centuries.

Keywords: climatology, trade-winds, tropics, sea navigation, Modern age.

\section{Contexto: revolución científica e Ilustración}

Los siglos XVII y XVIII reúnen algunos de los avances científicos más significativos de la civilización occidental. Para la ciencia climática este período va a resultar fundamental por los avances y mejoras que se producen en el instrumental meteorológico, en los métodos de análisis científico y en el conocimiento de las zonas climáticas terrestres. Ampliado muy notablemente el mundo conocido a partir de finales del siglo XV y desestimada la idea aristotélica de la inhabitabilidad de la zona tórrida, merced al conocimiento directo de las tierras americanas del ámbito intertropical, los siglos XVII y XVIII supondrán, para la disciplina climática, la formulación de ideas sobre el funcionamiento de los vientos, aspecto que se convertirá en el tema principal de estudio de científicos y pensadores de la época.

El siglo XVII es el siglo de la revolución científica, o mejor, de las pequeñas revoluciones en diversos campos de las ciencias que culminan con las decisivas aportaciones de Descartes y Newton. Se mejoran los sistemas de observación y el análisis de los fenómenos naturales y avanzan de manera determinante los métodos científicos en la búsqueda de respuestas a procesos físicos y naturales que superasen el providencialismo dominante. La apuesta renacentista por el ser humano se irá consolidando en el siglo XVII y culminará con el racionalismo ilustrado del XVIII. La propia expresión «revolución científica» para denominar a este período de aportaciones científicas fundamentales ocurridas en el momento central de la Edad Moderna se pone en entredicho 
al comprobar que estos avances se suceden en el tiempo, entre los siglos XVI y XVIII, sin suponer rupturas radicales sino continua suma de mejoras ${ }^{1}$.

Se desarrolla el empirismo, con las aportaciones de Bacon y Hume, que defenderán la experiencia sensorial como fundamento del conocimiento. Solo son reales los objetos y fenómenos particulares y la razón puede ordenarlos y obtener conclusiones inductivas a partir de ellos. Las ideas, para el empirismo, son copias de las impresiones. Por su parte, Descartes defenderá, en el marco del racionalismo, que solo la ordenación lógica del mundo que otorga el pensamiento permite comprender la realidad y establecer principios deductivos. Para ello, se potencia el método matemático de deducir a partir de unos pocos pero incontestables axiomas. Descartes defenderá la necesidad de la certeza mediante el uso de la duda y para ello debe partirse, según el filósofo francés, de realidades muy simples, a modo de verdades sobre las que no se pueda dudar, que sirven como punto de partida para la obtención de verdades más complejas (duda metódica). Desde la física, Newton es la gran figura de este período. La mecánica de Newton se convertirá en el modelo de lo que debía ser la teoría científica y tendrá efectos en la obra de científicos y filósofos posteriores.

En esta centuria se formulan, asimismo, un conjunto de leyes físicas de la máxima trascendencia, como la de los gases o Ley de Boyle, formulada de forma independiente por el físico irlandés Robert Boyle en 1662 y por el físico y botánico francés Edme Mariotte, en 1676, según la cual a temperatura constante el volumen de una masa de gas es inversamente proporcional a la presión ejercida. Newton enunciaría en sus Philosophiae Naturalis Principia Mathematica (1687) las famosas tres leyes del movimiento (inercia, aceleración y acción/reacción) que resultarán básicas para comprender el desplazamiento de los vientos en la superficie terrestre y, en especial, su segunda ley (ley de la aceleración) según la cual el movimiento de un cuerpo cambia bajo la acción de una fuerza al experimentar una aceleración en la misma dirección de la fuerza. Es interesante, asimismo, señalar los hallazgos relacionados con la luz y los fenómenos ópticos. Así, Descartes establecerá la ley de la refracción de la luz, estudiará también los espejos esféricos y las lentes y en su ensayo sobre Los Meteoros formulará una teoría para explicar la formación

1. REDONDO ÁlVARO, 198 (2008): 565-599. 
del arcoíris. En 1672, Newton propondrá la teoría corpuscular de la luz que junto a otras ideas y principios del físico inglés sobre la luz serán la base de su obra capital en esta cuestión, Optiks (1704). Unos años después, en 1678, Cristiaan Huygens dará un paso más en la explicación del fenómeno con su teoría ondulatoria.

Por su parte, el siglo XVIII conocerá el desarrollo de las ciencias matemáticas, física, química y naturales con aportaciones fundamentales para el avance posterior de las mismas. Así, Euler perfeccionó el cálculo infinitesimal y D'Alembert aplicó las matemáticas a la dinámica. Se idean y perfeccionan las escalas termométricas, esenciales para medir el calor en los procesos físico-químicos. Se realizan los primeros experimentos para el estudio de la electricidad, que encuentran en Benjamín Franklin a una figura principal. El científico estadounidense distinguirá, por primera vez, la electricidad positiva y la negativa y lleva a cabo una serie de experiencias que culminarán con la invención del pararrayos en 1752, amén de sus aportaciones sobre física de las nubes. Volta inventará la primera pila eléctrica compuesta de círculos de cinc y de cobre, aislados por un paño embebido en ácido sulfúrico diluido. Por su parte, la química se libera definitivamente de las concepciones alquimistas y se configura como una ciencia moderna. Boyle explica los cambios experimentados por los gases gracias a su constitución atómica, lo que le vale el apelativo de padre de la química científica. Joseph Priestly descubrirá un gas que favorecía la combustión, que denominó «aire flogisticado», siguiendo las ideas de la teoría del flogisto que había propuesto a mediados del siglo XVII el físico alemán Johann Becher. Esta sustancia sería bautizada, años después, como «oxígeno» por Antoine Lavoisier, que en 1785 enunciaría un principio, conocido como «ley de conservación de la materia»-descubierta asimismo, de modo independiente, por el químico ruso Mijaíl Lomonósov en 1748según la cual en toda reacción química la masa se conserva.

Por último, debe destacarse la aportación esencial del naturalista sueco Carl Linneo que propuso, en 1758, su conocido sistema de clasificación binomial de los reinos de la naturaleza, en lengua latina -la lingua franca de la ciencia-. El siglo XVIII es también el siglo de las teorías ambientalistas que influirán decisivamente en el desarrollo posterior de las disciplinas 
relacionadas con el territorio y su naturaleza. En opinión de Urteaga ${ }^{2}$, la influencia del clima en la explicación de las diferencias humanas, políticas y culturales del orbe terrestre fue un asunto común entre los tratadistas europeos del s. xviii, aunque nunca llegó a existir consenso completo sobre este aspecto. Tres campos centrarán lo esencial del ambientalismo o determinismo del factor climático en el s. XVIII. En primer lugar, la teoría etio-patológica, que hunde sus raíces en el legado hipocrático, según la cual las condiciones meteorológicas son uno de los factores determinantes de la salud humana y las diferentes características que aquellas presentan en la superficie terrestre permite establecer categorías de salubridad de los distintos ámbitos geográficos. En este razonamiento se encuentra el origen de la geografía médica. Junto a esta, la teoría de las razas, que explica la diversidad espacial de la especie humana en función de los rasgos que presenta el clima en cada lugar. Buffon en su Historia Natural (1749-1788), llega a afirmar que las razas no son una entidad esencial y definida sino el producto superficial y mudable del clima y los modos de vida. Buffon indica que el clima influye en la piel, el tipo de cabello y el color de los ojos. En la obra de Buffon se encuentra también la justificación del carácter civilizador del clima de latitudes medias y se apunta a la franja comprendida entre los $40^{\circ}$ y $50^{\circ}$ de latitud norte como la zona donde tuvo su origen el hombre y donde, en la actualidad, se encuentran las personas más hermosas y bellas y las mejor hechas. El tercer campo de reflexión del ambientalismo dieciochesco será la formulación de una serie de ideas histórico-políticas, que tratan de explicar la diversidad cultural de las diferentes regiones del mundo como efecto de los rasgos climáticos de cada una de ellas. En El Espiritu de las Leyes, Montesquieu afirmará que el clima gobierna temperamento y costumbres como argumento principal de la influencia del ambiente climático en la aparición y persistencia de formas culturales («las necesidades en los diferentes climas han dado origen a los distintos modos de vida»). Montesquieu dedica cuatro libros de esta obra (XIV a XVII) a establecer las relaciones del clima con las leyes en general, con las leyes «de la esclavitud civil», con las leyes de la «esclavitud política» y con las de la «servidumbre política». Otro filósofo ilustrado, Condorcet, defenderá asimismo el papel del clima como fuente de diferenciación cultural. Para el pensador francés

\footnotetext{
2.URTEAGA, XVIII/99 (1993).
} 
algunos pueblos han permanecido durante muy largo tiempo en los estadios tribales o pastorales, sin hacer progresos ni por su propio esfuerzo ni por el trato con otros pueblos civilizados, y esta apatía cultural se debe al clima. Aunque el ambientalismo y el determinismo climático serán ampliamente discutidos por numerosos filósofos de la época (Hume, Voltaire, entre otros), lo importante es destacar el nacimiento, en este momento, de una corriente de interpretación de las relaciones hombre-medio y de la diversidad de seres y lugares que mantendrá viveza hasta bien entrado el siglo Xx. En el fondo de los razonamientos de los ilustrados ambientalistas subyace la idea, defendida, como se ha señalado, en la Antigüedad, de la justificación de las latitudes medias como las zonas más aptas para el desarrollo de civilización.

\section{Avances en las ciencias atmosféricas en los siglos XVII y XVIII}

Como ya se ha indicado, el siglo XVII es el siglo de la aparición de los aparatos de medida de los elementos climáticos, que se irán perfeccionando en los siglos posteriores. Pero es ahora cuando se sientan las bases de lo que a partir del s. XIX se denominará climatología analítica o ciencia climática basada en el tratamiento de datos. En este sentido, debe destacarse la labor de Galileo Galilei, considerado como el fundador del método experimental y al que se le atribuye la construcción del primer termómetro científico; en realidad, Galileo inventó el primer instrumento capaz de medir los cambios de temperatura: el termoscopio neumático (1607). En 1639, Benedetto Castelli idea un primer pluviómetro que será perfeccionado en 1662 por Christopher Wren con un sistema de cubetas basculantes y mejorado en 1670 por Robert Hooke. Debe señalarse que el de Castelli no fue el primer pluviómetro de la historia, ya que los hay anteriores en países asiáticos, como Corea, China e India. Fue, eso sí, el primero creado en Europa. En 1641, Fernando II, Gran Duque de Toscana, construye el termómetro de bulbo de alcohol con capilar sellado; el instrumento estaba provisto de un tubo de vidrio con alcohol marcado con 50 grados, pero no utilizó el cero como un punto fijo. Por su parte, en 1644, tras llevar a cabo su famoso experimento, Torricelli construye el primer barómetro de mercurio. El primer higrómetro nació de la inventiva del físico francés Guillaume Amontons que lo presentaría en 1687 en la Academia de Ciencias francesa. Por último, el primer anemómetro para la medida de la velocidad 
del viento lo construye en 1667 Robert Hooke. De manera que, a finales del siglo XVII, se dispone del aparataje básico para la medición de los elementos climáticos principales. La puesta a punto de este instrumental meteorológico permitirá el desarrollo de los primeros embriones de redes meteorológicas. La primera de ellas, integrada por una decena de observatorios, fue instalada, a instancias del Gran Duque Fernando II de Toscana, por Luigi Antinori y funcionó entre 1654 y 1667. Será el germen de otras redes de observación que se irán ampliando y consolidando en los siguientes siglos.

En el estudio de los elementos atmosféricos, la medida de la presión atmosférica fue, sin duda, uno de los problemas importantes que tuvo que abordar la ciencia en el siglo XVII. Desde la época de Aristóteles habían perdurado dos ideas erróneas y generalmente admitidas. La primera de ellas era que el aire no pesaba y la segunda que no existía el vacío. Galileo rechazará ambas cuestiones y demostrará, mediante una serie de experimentos, que el aire pesa. En realidad, Galileo no llegó a entender del todo cuál era la naturaleza del aire y cómo actuaba la presión atmosférica. En 1643, su discípulo Torricelli resolvería el enigma, llevando a cabo una serie de experimentos en su laboratorio, llenando de mercurio un tubo de 1 metro de largo (cerrado por uno de los extremos). Invirtió dicho tubo sobre una cubeta llena de mercurio, de inmediato la columna de mercurio descendía por término medio, hasta una altura de 76 centímetros $(760 \mathrm{~mm})$. Torricelli interpretó que a esa altura se producía la influencia de la presión atmosférica. La comprobación en campo de este hecho la realizó, unos años después, en 1648, Florin Périer, por encargo de su cuñado Blaise Pascal, al medir la altura de una columna de mercurio a tres altitudes diferentes, durante su ascenso al Puy de Dôme. En este experimento se demostró que la presión atmosférica decrecía con el incremento de altitud, si bien ese descenso resultaba cada vez menos rápido.

En 1656, el alemán Otto von Guericke demostraría la existencia del vacío con su célebre experimento de los hemisferios de Magdeburgo, señalando que la presión atmosférica equivalía a un peso muy considerable. El experimento consistía en tratar de separar dos hemisferios metálicos, de unos $50 \mathrm{~cm}$ de diámetro, unidos entre sí por simple contacto, formando una esfera herméticamente cerrada, de la que se extraía el aire con una bomba de vacío, por cierto, inventada por el propio von Guericke. Para facilitar el cierre hermético de las semiesferas metálicas se disponía de un aro de cuero que se 
colocaba entre las superficies que se tocaban. Cada hemisferio disponía de varias argollas para pasar cuerdas o cadenas por ellas y así poder tirar hacia los lados opuestos. Dos tiros de caballos se encargaron de ello, dando gran espectacularidad al experimento, lo que causó gran asombro entre el público asistente. Cuentan las crónicas que tras varios intentos, los caballos lograron separar los hemisferios. En los hemisferios solo actúa la presión atmosférica puesto que al extraer el aire no hay presión en el interior. El aire atmosférico ejerce presión sobre los hemisferios y, si la superficie de los mismos sobre la que actúa la presión es suficientemente grande, se necesita una fuerza bastante considerable para separarlos.

El siglo XVIII traerá, por su parte, una serie de avances de gran importancia para las ciencias atmosféricas, que resultarán fundamentales para consolidación de la meteorología y climatología como disciplinas científicas. Estos avances se refieren a la mejora en el instrumental y sistema de medidas de elementos climáticos, al establecimiento de las primeras redes de observación, a la explicación de fenómenos atmosféricos y mecanismos de circulación general y, por último, a la interpretación del reparto de climas en la superficie terrestre y a la influencia de estos en los seres humanos. En efecto, desde mediados del siglo XVII y en las primeras décadas del xviii una serie de investigadores contribuirán al perfeccionamiento y sistematización del instrumental y unidades de medida meteorológicas. Así, por ejemplo, antes de que concluya el siglo XVIII se dispone de las cuatro escalas termométricas más difundidas, esto es, las de Fahrenheit (1724), con importante perduración aún en el ámbito anglosajón, Reamur (1730), Celsius y la escala centígrada, estas dos últimas tenidas erróneamente por equivalentes. Hay que recordar que Anders Celsius propuso en 1742 el uso de una escala donde se atribuía el valor $100^{\circ}$ al punto de fusión del hielo y $0^{\circ}$ al de ebullición del agua, es por tanto inversa a la denominada «centígrada». Esta última sería difundida en Suecia tres años después por Linneo, aunque ya había sido manejada en esta posición por Jean-Pierre Christi en Francia en 1743, en su famoso «termómetro de Lyon» donde asignó el valor $0^{\circ}$ al punto de fusión del hielo y $100^{\circ}$ al de ebullición del agua.

El resultado de la puesta a punto del instrumental meteorológico favorecerá el desarrollo de observaciones atmosféricas sistemáticas durante la segunda mitad del siglo XVIII y, sobre todo, en el último cuarto de esa centuria, cuando 
se proponen los primeros embriones de redes o servicios meteorológicos. En la creación y consolidación de estas redes de observación meteorológica jugarán un papel destacado las Sociedades de Medicina, debido a la relación entre el desarrollo de enfermedades y las condiciones atmosféricas de un territorio. Esta relación encontrará argumento filosófico dentro del ambientalismo que impulsarán algunos ilustrados de la época (vid. infra).

El propio Immanuel Kant, autor clave en la evolución de la disciplina climática en el siglo de la Ilustración, será consciente de esos avances, como se manifiesta en la serie de científicos y sus obras a las que se referirá ampliamente el filósofo en el apartado dedicado a la «Atmósfera» de su Geografía Física ${ }^{3}$.

\section{La explicación de los vientos alisios: la gran cuestión}

Un tema principal para la filosofía natural y la física durante los siglos XVII y XVIII fue el viento. No en vano, como señala, Pelkowsky ${ }^{4}$, en el siglo XVII se consolidará la «pneumática», arte creado en el período helenístico, encargado de estudiar la naturaleza, el peso y la presión del aire y sus efectos. Inicialmente organizada en torno a dos innovaciones instrumentales ocurridas en este período (el tubo de Torricelli y la bomba de vacío), irá ampliando su campo de estudio en la centuria siguiente e incorporando el análisis del termómetro, los fenómenos térmicos y meteorológicos, el estudio y la naturaleza del sonido y hasta los tipos y las características de los vientos. La Enciclopedia Británica incluiría la voz «pneumática» en su primera edición de 1768, con la explicación de todos estos aspectos, pero con estudio detallado de las cuestiones relativas al viento.

El origen del viento y sus movimientos animaron un intenso debate de propuestas entre los filósofos y físicos de la época, que deseaban superar las ideas aristotélicas sobre este elemento climático. Recordemos que Aristóteles, en Los Meteorológicos (s. IV a. C.) relacionaba los fenómenos atmosféricos con la actividad del Sol que originaba dos tipos de «exhalaciones»: una caliente y seca producida por el fuego que se levanta de la tierra; otra caliente y húmeda (vapor) producida por el agua, la cual se levantaba de la parte acuosa de la tierra. La exhalación húmeda no se da sin la seca, ni la seca sin la húmeda.

3. Olcina Cantos, 34/2 (2014b): 119-162.

4. PelKoWSKY, 5/14 (2006): 24-30.

Revista de Historia Moderna, n. ${ }^{o} 35$ (2017) (pp. 8-45) | ISSN-e: 1989-9823 | ISSN: 0212-5862 
Los diferentes meteoros eran formados por ellas. Las «evaporaciones» sufrían transformaciones debido a los cambios de temperatura, a la variación del calor del suelo por la proximidad a la esfera de fuego. De la «evaporación» seca se producía el trueno, el rayo, los vientos, los terremotos, los cometas y otros fenómenos ardientes. Del «vapor» húmedo se desarrollaban las nubes, la lluvia, la nieve, el granizo, la niebla, el rocío y las heladas (Libro II, cap. 4). Para Aristóteles la causa del viento es clara: «El Sol hace cesar e impulsa a los vientos. Consumiendo la exhalación los hace cesar y con la rapidez de la desecación impide que se formen» (Libro II, cap. 5). Aristóteles otorga a la zona tórrida el rasgo de ser una región principal de producción de vientos del sur hacia el norte debido a la cercanía del Sol e incluso señala que dado que «esa región es mucho más amplia y más abierta, el viento del sur es más grande, más fuerte y más cálido que el del norte; y llega más aquí que este allí» (Libro II, cap. 5). Este aspecto que es causa, además, de la inhabitabilidad de la región tórrida, será rechazado a partir del siglo XV y, especialmente, en el siglo XVI, tras el descubrimiento del Nuevo Mundo y la constatación de la existencia de vida humana en la zona tórrida del planeta. Así lo manifestarán varios cronistas de Indias en sus escritos y, particularmente, el padre Acosta en su Historia Natural y Moral de las Indias ${ }^{5}$.

La búsqueda de la causa última del movimiento de los vientos en la atmósfera terrestre tenía por objeto principal, en la Edad Moderna, la explicación de los vientos constantes del ámbito intertropical (alisios) que recibían, por lo común, la denominación de «brisa» constante. En 1685 se abrió un debate sobre la circulación general de la atmósfera que comenzó en el seno de la Royal Society de Londres. No era de extrañar esta preocupación por las cuestiones climáticas y, específicamente, por la circulación de los vientos, en un país con tan destacada vocación y actividad marítima. El obispo escocés George Garden promovió con entusiasmo, ese año, la idea de Galileo de que los vientos alisios «se quedaban retrasados» porque el aire, debido al caldeamiento del Sol, se torna más ligero, se levanta y pierde contacto con la superficie terrestre. En ese contexto, el científico francés Edme Mariotte repetirá también la hipótesis de Galileo, pero explicó la tendencia del viento

5. Olcina Cantos, XVIII/478 (2014a).

Revista de Historia Moderna, n. 35 (2017) (pp. 8-45) | ISSN-e: 1989-9823 | ISSN: 0212-5862 
a acercarse al ecuador desde el noreste y el sureste como consecuencia del movimiento del Sol entre los equinoccios ${ }^{6}$.

Recordemos que unas décadas antes, Galileo había explicado los vientos alisios como un efecto del desajuste en el envoltorio gaseoso de la Tierra en relación a la velocidad de rotación de la misma. La Tierra gira más rápido en las latitudes más próximas al ecuador, de manera que el aire y el agua se quedan retrasados y cualquier observador terrestre observará un flujo dirigido hacia el oeste. Por el contrario, el flujo de viento dirigido hacia el este en las latitudes medias está causado por el mecanismo opuesto: la Tierra gira más lentamente y el aire y el agua «se adelantan». Similar argumento sería utilizado por Johannes Kepler para explicar el movimiento hacia el oeste de las aguas en los océanos tropicales. Tanto para Galileo como para Kepler la rotación de la Tierra no solo explicaba los vientos alisios, sino que los vientos alisios eran ante todo una manifestación de la propia rotación de la Tierra ${ }^{7}$.

Tras las críticas a las ideas aristotélicas sobre el mecanismo generador de los vientos en el siglo XVI, será Francis Bacon, en la centuria siguiente, el que rechace de manera más intensa los argumentos clásicos sobre el funcionamiento de los vientos en sus reflexiones sobre filosofía natural. En su Historia Natural y Experimental de los vientos (1622), Bacon ofrece una nueva explicación para entender el funcionamiento de los vientos generales, en esencia los vientos constantes del ámbito intertropical:

«Hay pocos fenómenos observados sobre los vientos generales; no es sorprendente, ya que estos vientos se encuentran principalmente dentro de los trópicos, donde precipuamente se encuentran los lugares condenados por las antiguos por inhabitables. [...] Es un hecho cierto que un viento constante de Este a Oeste, llamado «Brisa» por los navegantes, sopla constantemente entre los trópicos; pero su causa es dudosa. Puede ser debida a esto: que el aire, como habíamos notado previamente, se mueve en la dirección de los cielos, aunque menos perceptiblemente fuera de los trópicos, a raíz de los menores círculos allí, mientras que es evidente dentro de ellos, donde los círculos son más grandes. Otra razón puede ser esta: que todo aire es dilatado por el calor, y, debido a esta dilatación no puede ser contenido en el mismo espacio, el aire contiguo es forzosamente impulsado, de tal forma que crea la brisa constante; mientras que el Sol mantiene su curso. Pero esta dilatación

6. PERSSON, 3 (2006): 17-42.

7. Ibídem. 
ha de ser más considerable dentro de los trópicos, donde el Sol tiene mayor poder; fuera de ellos, es apenas imperceptible».

Un aspecto importante en la deducción de Bacon es determinar si estas «brisas» se producen por la noche, puesto que la rotación del aire continúa por la noche pero el calor del Sol no. Al respecto, señala que «es seguro que esta brisa no sopla durante la noche, pero sí en la mañana y algún tiempo después del amanecer». Y sobre la trayectoria de esta «brisa» indica que «si el aire participa en el movimiento del cielo, no solo que el viento del este es concurrente con el movimiento del aire, y el viento del oeste se opone a ello». Y concluye: «La brisa constante de que hablamos (que puede ser un movimiento del aire) no es del este, sino del nordeste». Bacon otorga, por tanto, un papel importante al movimiento del Sol en la explicación de la propia trayectoria de los vientos y a los procesos de dilatación que experimenta el aire calentado por la radiación solar como causa del movimiento inicial ${ }^{8}$.

En relación con esta cuestión es necesario referirse a la formulación de la primera visión de conjunto sobre la circulación atmosférica general que tiene argumento principal en el funcionamiento de dicha circulación en el ámbito intertropical. Este esquema se debe al prestigioso científico inglés Edmund Halley. En un artículo de 1686, básico en la historia de la ciencia climática ${ }^{9}$, el sabio inglés indaga sobre la causa última de los vientos alisios (trade winds) y de los monzones. Realiza una descripción exhaustiva de las áreas planetarias donde soplan estos vientos principales de la circulación atmosférica sobre la superficie terrestre y de los rasgos estacionales de los mismos. Esboza el primer mapa de vientos del ámbito intertropical indicando la dirección de soplo de ambos vientos regulares que luego será referencia en planisferios elaborados por diferentes autores en los siglos XVII y XVIII (Figura 1).

Halley indaga en su ensayo la causa última que origina el movimiento y dirección de estos vientos y afirma que frente a otros autores que indican la importancia de la rotación terrestre en la explicación del soplo de los alisios

8. El Tratado sobre los vientos de Francis Bacon, de 1622, es un ensayo detallado sobre este elemento climático que incluye una clasificación de vientos y su explicación causal. En él se contrastan las opiniones expresadas por autores clásicos (Aristóteles y Plinio el Viejo) y modernos (José de Acosta). Puede consultarse on line: http://www.sirbacon. org/naturalhistorywinds.htm

9. HALLEY, 16/179-191 (1686): 153-168. 


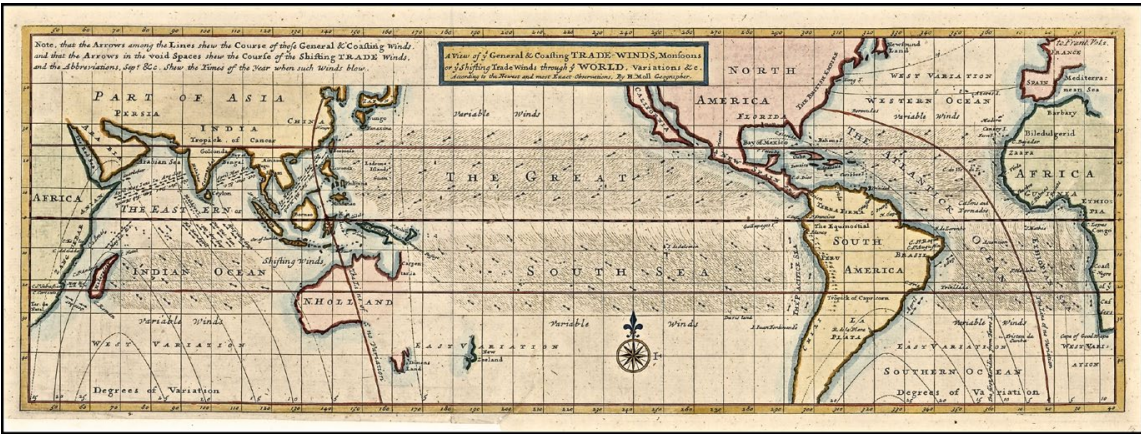

Figura 1. Planisferio terrestre con indicación de la zona afectada por el soplo de los vientos alisios (trade winds) en sombreado, dentro del ámbito intetropical. Fuente: «A View of ye General \& Coasting Trade-Winds, Monsoons or ye Shifting Trade Winds through ye World, Variations \&c...», elaborado por Hermann Moll (ca.1730).

y los monzones, la verdadera fuente o fuerza motriz que origina estos vientos constantes en el ámbito intertropical es el calor originado por la radiación solar en su movimiento diario y estacional. Ese calor cuasi constante es el causante de un proceso de rarefacción del aire en estas latitudes (un aire «sobre treinta veces más ligero» en las proximidades del ecuador) que favorecería el movimiento de ese aire desde las áreas situadas en las líneas de trópico (Cáncer y Capricornio) hacia la línea equinoccial. Y ello, según Halley, siguiendo las leyes de la estática según las cuales el aire se mueve siempre desde las áreas con menor «enrarecimiento» (aire más pesado) a aquellas más enrarecidas; esto es, el aire se mueve de las zonas menos cálidas del ámbito intertropical a las zonas más cálidas.

La curvatura de estos vientos alisios hacia el SW (hemisferio norte) y hacia el NW (hemisferio sur) se debería al propio movimiento diario del Sol (Este a Oeste) y la diferente recepción de calor en los mares del ámbito intertropical que ello supone a lo largo del día. Por así decir, el viento se iría «moviendo» hacia el W siguiendo al Sol en su movimiento diario. Para justificar el carácter «constante» de estos vientos alisios, Halley señala que «se forma un viento general del este, que se imprime a todo el aire de un vasto océano, con las partes impeliéndose una a otra, para mantener el movimiento hasta el próximo regreso del Sol, recuperándose entonces tanto movimiento como se había perdido, y perpetuando de ese modo el viento del este». Se 
transmite, indirectamente, la idea de que los vientos alisios perderían fuerza durante la noche, cuando el Sol desaparece, que no es real.

Esta misma causa primera (calor) explicaría el movimiento de los monzones en el sector septentrional del océano Índico. La diferencia aquí es la estacionalidad del calor en una zona continental que origina un flujo del SW en verano dirigido hacia el trópico, que se torna del NE en invierno, debido no solo al desplazamiento anual de la fuente de calor (Sol) sino al enfriamiento que se registra sobre el espacio continental en esos meses fríos del año. Halley señala que existiría una «dificultad» para explicar esta alternancia estacional de vientos en el océano Indico sur puesto que aquí no se produciría, debido a su carácter marítimo, y el monzón soplaría todo el año del SE.

Por último, Halley explica las alteraciones que estos flujos de viento constantes experimentan sobre superficies continentales dentro del ámbito intertropical. Dos causas principales, según Halley, originan esta modificación en el sentido de flujo: la naturaleza del suelo y la posición de las altas montañas. Estos dos factores alteran el enrarecimiento del aire allí donde afectan y llegan a causar tanto ascensos del aire, que origina lluvias («raines» en el texto de Halley), como formación de calmas sobre el océano en la misma línea ecuatorial, o por otro lado flujos de viento del W en el golfo de Guinea. Halley señala que los flujos del Este (alisios) o del W (golfo de Guinea) establecen un sistema en equilibrio. Además el peso del aire en estas zonas que, según Halley, se ve disminuido por el soplo de estos vientos contrarios, impide que el aire mantenga todo el vapor de agua que recibe y lo deja caer en las copiosas precipitaciones que allí se generan. Halley esboza, de este modo, una explicación -incompleta- de las precipitaciones abundantes del área ecuatorial.

Su explicación sobre la causa principal de la circulación de vientos en el ámbito intertropical es una aproximación de enorme interés por el importante papel que Halley otorga al Sol como fuente de calor y motor de los movimientos atmosféricos. No obstante, adolece de un error básico al despreciar la incidencia de la rotación terrestre y de las diferencias de velocidad angular del viento en las diferentes latitudes terrestres como agente principal de encurvación de flujos aéreos y de establecimiento, en suma, de la dirección de los vientos (Figura 2). 
En su ensayo no llega a proponer un modelo de circulación atmosférica general válido para todo el globo. Se ha señalado, erróneamente, que Halley defiende la existencia de una célula única de circulación atmosférica entre latitudes ecuatoriales y polares: la denominada «chimenea ecuatorial» con vientos alisios enfrentados en la línea equinoccial, ascendiendo en esas latitudes ecuatoriales y desplazándose en altitud hacia los polos para descender allí por efecto de la mayor densidad del aire enfriado a lo largo de su desplazamiento hacia latitudes polares. Pero en ningún momento de su ensayo se lleva a señalar este extremo, puesto que como reza el propio título de su trabajo, Halley se limitó a dar explicación al mecanismo de soplo de los alisios y los monzones en latitudes tropicales, entre $30^{\circ}$ norte y sur. De manera que la idea de un modelo de circulación unicelular, basado en la idea de una «chimenea» originada por el calor ecuatorial nunca existió en la propuesta de Halley, que únicamente refiere su propuesta al ámbito intertropical:

«Por lo tanto, el aire al norte y al sur, más denso que en el centro, ha de tender de ambos lados hacia el ecuador: este movimiento del viento, combinado con el anterior, del este, dilucida todos los fenómenos de los vientos alisios, que indudablemente soplarían durante todo el año, si toda la superficie del globo constara de mar, como sabemos que lo hacen en los océanos Atlántico y Etiópico» ${ }^{10}$.

Una figura destacada en la centuria del XVII en la descripción de cuestiones geográficas y estrictamente climáticas es Bernand Varenio, que en su Geografía General, dedicará dos capítulos (caps. XX y XxI) al viento ${ }^{11}$. En el primero de ellos Varenio señala que son varias las «causas de los vientos». Siguiendo las ideas de los clásicos y apoyándose en su coetáneo Francis Bacon ${ }^{12}$, proporciona la siguiente descripción sobre el origen del viento:

«La causa general y principal es el Sol mismo, el cual, mediante sus rayos de fuego, enrarece y atenúa el aire, sobre todo el que se encuentra debajo de él.

10. Vid. Halley, op cit. p. 166.

11. Olcina CANTOS, 2016: 815-852.

12. Vid. Francis Bacon Historia naturalis et experimentalis de ventis, Londini: In officina Io. Hauiland, impensis Matthaei Lownes \& Guilielmi Barret, 1622. Se trata de un trabajo de 285 páginas incluido dentro de la obra de este mismo autor «Historia naturalis et experimentalis ad condendam philosophiam, sive, Phaenomena vniuersi quae est Instaurationis magnae, pars tertia». 

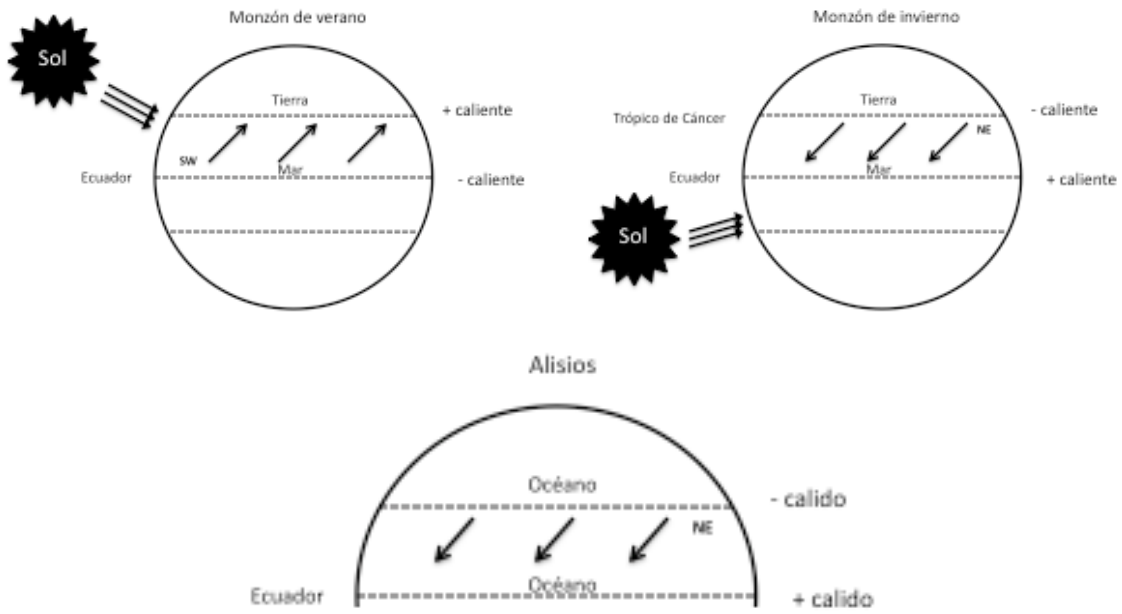

Figura 2. Explicación del mecanismo de circulación de los monzones y de los vientos alisios según E. Halley (1686).

El aire enrarecido ocupa un mayor espacio, lo que hace que el aire empuje hacia adelante el aire contiguo; y el Sol, teniendo un curso circular del este al oeste, hace que la presión se ejerza hacia el oeste, como parece ser el caso en la mayoría de los lugares de la zona tórrida y en todos los alrededores, donde reina continuamente sobre el mar un viento del este; el aire enrarecido presiona hacia el oeste dentro de los trópicos. Existe, efectivamente, una presión por todos lados; pero el aire apenas es admitido en los otros puntos porque la presión no es tan grande como del lado de oeste, dado que el Sol se mueve hacia ese lado» (Cap. XX, prop. X).

Y junto al Sol, Varenio enumera seis procesos más que «ayudan» a esta causa principal:

- las exhalaciones del mar y de la tierra que contribuyen a rarificar el aire

- la rarefacción o atenuación de las nubes

- la fusión del hielo y la nieve

- el ascenso de la Luna y los astros

- la condensación y rarefacción del aire por el frío y el calor

- el descenso de las nubes que presiona el aire inferior 
No es extraño que, entre las noticias que Varenio pudo recoger de los navegantes en la ciudad de Ámsterdam, la justificación del soplo de esos «vientos constantes» del Este que los viajeros encontraban en sus travesías oceánicas ocuparan un protagonismo principal, que luego reflejó en estos apartados dedicados al viento en su Geografía General.

Varenio aporta su explicación del soplo constante de estos vientos del Este («General Winds»): «...el Sol es la principal causa que enrarece al aire del este hacia el oeste; por consiguiente, el aire es más presionado del lado del oeste. Este movimiento no puede ser impedido, a no ser por una gran cantidad de exhalaciones o nubes en la parte oeste, lo cual no es frecuente»; y añade:

«Los naturalistas señalan tres causas de este viento general (puesto que los antiguos no tenían ningún conocimiento ni siquiera de la zona tórrida). Algunos piensan que el movimiento del Sol del este al oeste es la causa, porque enrarece el aire por donde pasa, y que esta rarefacción sigue al Sol que empuja el aire delante de sí. Otros, es decir, los que suponen que los cielos son inmóviles y que la Tierra gira, son del parecer que el viento general viene del movimiento de la Tierra del oeste al este, y que el aire gira con ella, pero no tan rápido como nosotros; y por consiguiente, dicen que vamos contra el aire, o el aire contra nosotros del este al oeste. La tercera causa, nueva, fue imaginada por Descartes...donde trata de mostrar que ese movimiento del aire es causado por la luna también, como las mareas...»

Entre estas tres causas, Varenio se decanta por la primera y rechaza la segunda «porque varios copernicanos la han rechazado, y no podemos aducir ninguna razón de por qué no soplaría sino entre los trópicos, y no más allá» ${ }^{13}$.

Señala Varenio dos ámbitos mundiales donde los vientos del Este soplan todo el año: el océano entre Sudáfrica y Brasil (Atlántico sur) y el océano entre América y las Islas Orientales (Pacífico); en otros ámbitos oceánicos del planeta estos vientos no merecerían el calificativo de «vientos generales», por ejemplo entre Sudáfrica y las Indias orientales, debido en este caso a la aparición del fenómeno de monzón, o en el Atlántico norte en África intertropical debido a la existencia de un área de calmas en el golfo de Guinea.

13. En las ediciones de la Geografía General preparadas por James Jurin (1712, 1736), se incluiría, en este apartado, la referencia a la aportación realizada por Edmund Halley en su célebre artículo de 1686 sobre las causas de los vientos alisios y los monzones. 
Varenio dedica un extenso apartado del capítulo XXI de la Geografía General a la explicación de los vientos periódicos, que engloba bajo el concepto común de «monzones» (Cap. XXI, proposición 3). Señala que algunos de estos vientos monzónicos cambian de dirección dos veces al año en diferentes partes del mundo, pero señala que no con la misma fuerza. Incluye una relación de aquellos lugares del mundo donde son importantes los vientos periódicos. Se trata de un listado amplio de áreas terrestres que registran vientos constantes en una época del año (vientos periódicos), pero que realmente no corresponden a lo que se entiende como circulación de monzón propiamente dicha, aunque Varenio emplee este concepto ${ }^{14}$. Se comprueba en este apartado la recopilación de información realizada por el geógrafo alemán, en su estancia en la ciudad de Ámsterdam, a los navegantes que hacían las rutas de comunicación fluvial entre Europa y Asia, África y América:

- El Atlántico intertropical, donde los vientos soplan del Nnorte de octubre a enero («la mejor época para navegar de Europa a la India»)

- En Cabo Verde, donde soplan del Sur en verano y originan «el invierno lluvioso» allí.

- En el cabo de Buena Esperanza, donde sopla viento del Noreste en septiembre.

- $\quad$ El reino de Patani (Sudeste Asiático) que registra vientos del Noreste en invierno y del Este en verano.

- Sumatra, donde el monzón cambia en noviembre y diciembre.

- Las islas Azores, que registran un viento periódico del Sur en agosto.

- La región del Congo, con vientos del Norte y Noroeste de marzo a septiembre, y del Sur y Sureste de septiembre a Marzo.

Sobre las causas de estos vientos periódicos, Varenio señala que son diversas en cada área del mundo, pero apunta tres fundamentales. Dos de ellas principales: la fusión de la nieve de las montañas y la disolución de las nubes en áreas de hemisferio norte y sur. Ambas, según Varenio, están originadas por la

14. Tanto en este detallado apartado sobre los vientos periódicos como en el dedicado a los vientos constantes del Este en el ámbito intertropical se puede apreciar la importante labor de recopilación de información realizada por Varenio a los navegantes que hacían las rutas de comunicación fluvial entre Europa y Asia, África y América. 
propia radiación solar, que incide más en un hemisferio que en otro en cada mitad del año. Cuando el Sol está en el hemisferio norte, soplarían desde el norte y viceversa. Además, indica una tercera causa de los vientos periódicos: el mismo movimiento de la Luna y la fase lunar en que se encuentre en cada momento del año que «pueden causar un cambio en esta cuestión» (Cap. XXI, prop. 3).

Junto a las áreas mundiales donde soplan esos vientos periódicos, Varenio afirma con rotundidad que la región del mundo donde «los monzones son más famosos» es el océano Índico, entre África y la India, puesto que aquí el cambio en la dirección de los vientos tiene lugar cada seis meses. Se describen, a continuación, con detalle los lugares de Asia meridional y oriental (entre las islas del Índico hasta Japón) donde se observa este mecanismo de vientos periódicos, indicando el momento exacto del año en el que se produce el cambio en el rumbo de los vientos. En este espacio del mundo los vientos soplan, según indica Varenio, del oeste en una parte del año (verano) y del este en la otra (invierno) ${ }^{15}$. Varenio afirma que los vientos periódicos más constantes se encuentran sobre el océano, pero no ofrece una causa para la explicación del monzón en el sur de Asia porque es consciente de las diferencias existentes en este extenso espacio terrestre lo que impide una única causa para justificar su soplo y su rumbo. Y asimismo, señala la necesidad de disponer de un mayor número de observaciones de varios años para poder sacar conclusiones sobre su funcionamiento, «así como del mejor conocimiento en la estación invernal, de las lluvias, las nieves y de las montañas en esos lugares desde los que esos vientos comienzan a soplar». De manera que estos vientos del monzón asiático quedarían justificados por la causa general ofrecida por Varenio para comprender los vientos periódicos que se ha señalado con anterioridad (vid. supra).

En el último tercio del siglo XVII se desarrollará una nueva, e ingeniosa pero incierta, propuesta de explicación de los vientos alisios. Se trata del trabajo publicado por el Dr. Martin Lister el año 1684 y editado, asimismo, en la prestigiosa Philosophical Transations. Según su teoría los trade winds estaban causados por el «constante aliento de las algas» (sargessa, lenticula marina)

15. La circulación genuina del monzón en el sur de Asia sopla del Noreste (NE) en invierno y del Suroeste (SW) en verano. 
en el océano» y su dirección estaba originada por el movimiento general del mar en la zona tropical.

Ya en la centuria del s. XVIII se encuentran nuevos intentos de explicación de la circulación de los vientos en la atmósfera terrestre y se proponen nuevos razonamientos para justificar su génesis y movimiento. Resulta muy notable el trabajo de George Hadley, publicado en 1735, donde propondrá un modelo de circulación atmosférica general, cuya conclusión básica es que en el hemisferio norte el flujo de vientos es norte-sur y a la inversa sucede en el hemisferio austral. Particular interés muestra Hadley en justificar el rumbo de los alisios; considera que la incurvación hacia el oeste de los alisios resulta de la incidencia de la rotación terrestre y del incremento de velocidad lineal a medida que se pierde latitud.

En 1771, la primera edición de la Enciclopedia Británica incluiría, dentro de la voz «pneumatics» (neumática, estudio del aire), una explicación del movimiento de los vientos regulares que soplan en el ámbito intertropical, los denominados trade winds. En dicho texto se indica, recogiendo plenamente las ideas de Hadley, que los vientos alisios están originados por dos causas (térmica y dinámica): "One is the great heat of the equatorial region, by which alone would be produced a constant north or south wind. The other is the diurnal revolution of the earth, which would cause a perpetual tendency of the air in these warm regions from east to west ${ }^{16}$. De la combinación de estas dos causas resulta la formación de los vientos alisios (trade winds) que soplan de forma constante desde el SE o desde el NE. No obstante, para justificar la dirección que adquieren los alisios en algunas zonas costeras del planeta, donde se convierten en vientos de norte o de sur, según el hemisferio, se señala como origen el menor protagonismo que puede tener una de estas dos causas en esos territorios o bien la influencia ejercida por la presencia de factores locales como altos relieves.

Poco conocidas, pero fundamentales en el contexto de evolución de la disciplina climática, son las aportaciones sobre los vientos del filósofo Emmanuel Kant. En efecto, el viento y, en general, el movimiento del aire es el elemento climático más importante para Kant a tenor de la extensión que le dedica en la Geografía Física (1802) y en algunos escritos previos sobre cuestiones de

16. Vid. Enciclopaedia Británnica 1771, vol.3. p. 499. 
la Naturaleza. Kant se interesa por conocer los rasgos de los vientos que eran conocidos en su época y por explicar su causa última. Y es en este último aspecto donde mejor se pueden apreciar las aportaciones del filósofo alemán a las ciencias meteorológica y climática. Es lo que algún autor ha denominado la «anemología kantiana» ${ }^{17}$.

No debe extrañar, por tanto, que el viento sea el elemento climático que más páginas ocupe en la Geografía Física de Kant. Recordemos la importancia de este factor atmosférico para la navegación marítima en esta centuria ilustrada, que había favorecido la aparición de tratados de navegación en los que la descripción de los diferentes tipos de viento y, en ocasiones, la explicación de su origen ocupaba buen número de páginas. Dentro de la ciencia española ilustrada destacan, al respecto, los trabajos de los eruditos ilustrados y marinos Jorge Juan y Antonio de Ulloa, que llevarán a término la famosa expedición a América entre 1736-39 y publicarán sus resultados unos años después (1748); este último será citado expresamente por el propio Kant en su tratado. En efecto, las Observaciones Astronómicas y Físicas (1748) de ambos marinos contienen varias anotaciones sobre los vientos encontrados durante la expedición ${ }^{18}$. Las Noticias Americanas de Antonio de Ulloa (1792), están repletas de referencias a los vientos y sus características e, incluso, indica la existencia de la «brisa» (alisios) al sur de Canarias y en las proximidades de la línea equinoccial, que permiten la navegación rápida en los océanos del ámbito intertropical ${ }^{19}$. Por su parte, el Compendio de Navegación de Jorge Juan (1757) incluye diversas referencias sobre la importancia del conocimiento del rumbo de los vientos para las rutas y se relacionan los rumbos principales de los vientos ${ }^{20}$.

A todo ello se une que el estudio y medición del resto de elementos climáticos tiene aún en ciernes en el siglo XVIII, el desarrollo de aparataje y de unidades de medida que permitan un estudio más detallado de temperaturas, precipitaciones, humedad o presión atmosférica. En la Geografía Física hay

17. PELKOWSKI, 2004.

18. Vid. JuAn y Ulloa, 1748.

19. Vid. Ulloa, 1792.

20. Vid. JUAN, 1757. 
comentarios y descripciones de estos elementos climáticos, pero se incluyen muy pocos datos con unidad de medida de ellos.

Kant utiliza una comparación para definir el viento al señalar que el aire en movimiento es como «una corriente de agua», pero no es muy preciso al mantener esta comparación y señalar que «como el mar está limitado por la dirección de la tierra firme y las montañas», cuando hay muchos más factores que pueden condicionar la acción del viento. Sí que acierta, por el contrario, al indicar que el choque de dos corrientes de viento opuestas, como ocurre en el mar, "produce torbellinos», adelantando lo que décadas después se consolidará como los mecanismos dinámicos de inestabilidad atmosférica.

Para el filósofo alemán hay tres principios principales, de raigambre exclusivamente térmica, que explicarían el funcionamiento del viento:

- El primer principio señala que el viento se mueve siempre de sectores más fríos a zonas calentadas y la duración del soplo del mismo depende de la misma duración del calentamiento existente en ese lugar. Está adelantando Kant la explicación de las brisas de mar y de tierra, generadas por diferencias de temperatura y presión atmosférica que se fijará definitivamente como causa de las mismas, a comienzos del siglo Xx, en el primer teorema de V Bjerknes. Kant, sin embargo, no otorga protagonismo a la presión atmosférica en la formación de las brisas y cede a las diferencias de temperatura entre mar y tierra en los espacios de costa el papel principal de su génesis. En efecto, para Kant este desplazamiento del aire hacia las zonas más cálidas se produce porque los sectores más calientes «ceden ante esta corriente de viento, al ser más ligera que la del aire frío».

- El segundo principio que explica el movimiento del aire tiene que ver con la propia densidad del mismo. Para Kant, si una zona se enfría poco a poco el aire situado sobre ella se contrae y deja espacio para que sea ocupado por aire más cálido. No es un razonamiento del todo correcto, puesto que el movimiento del aire, sea frío o cálido, no depende solo de principios térmicos, sino que la presión atmosférica juega un papel básico a la hora de explicar estos desplazamientos. 
Con estos dos principios, que Kant había expuesto ya en su «Ensayo sobre la teoría de los vientos» (1756), el filósofo justifica el mecanismo de los vientos de mar a tierra (brisa diurna) y de tierra a mar (brisa nocturna) que se dan en zonas de costa o en islas. El principio que justificaría el soplo de este circuito de viento periódico sería, para Kant, simplemente térmico.

- Por último, como tercer principio del movimiento del viento, Kant señala que la formación de nubes de tormenta o su propio funcionamiento interno está en relación con vientos «sulfurosos y minerales que llegan a fermentar» y generan «espantosas lluvias»; por su parte, una vez formada la tormenta, con aguaceros fuertes o granizo se originan vientos «que pueden llegar a ser muy violentos». La primera parte de su razonamiento se ha demostrado errónea, pero la segunda, justifica la formación de remolinos o torbellinos en una nube de desarrollo vertical.

Pero, sin duda, el apartado más destacado dentro del análisis del viento en la Geografía Física es el que hace alusión a la explicación de los vientos alisios (trade winds). Kant acepta esta denominación pero no explica su origen ${ }^{21} \mathrm{y}$ señala que la causa de que entre los trópicos sople «un viento este» de forma casi permanente es térmica: «Este -viento- no se origina a partir del aire que se retrasa, el que, como la Tierra gira de la tarde a la mañana, queda retrasado y resiste en la dirección opuesta, sino del calentamiento progresivo del día a la tarde por el Sol alrededor de la Tierra».

En esta explicación, Kant recupera el primer principio sobre el soplo del viento que había señalado en apartados anteriores (vid. supra) según el cual el viento se desplaza desde las zonas más frías a las más cálidas. De manera

21. Pelkowski señala que la expresión «trade-winds» no significaría, como se entiende habitualmente, «viento del comercio» en referencia a los vientos del ámbito intertropical que ayudaban a los veleros a desplazarse desde Europa al Nuevo Mundo en su travesía por el océano Atlántico, sino que hace proceder el vocablo «trade» del verbo «tread» que se traduce por «pisar» $\mathrm{o}$ «hacer sendero». Por su parte, el vocablo francés «alizés» que se emplea para denominar a los vientos alisios significaría «estar unido», «ser plano o liso». No obstante, en el siglo XVIII, tanto en la Cyclopaedia (Londres, 1728) como en la Encyclopedia Britannica (Edimburgo, 1771), la explicación de los vientos alisios maneja la expresión «trade winds» en la acepción más comúnmente aceptada de «vientos del comercio». PELKOWSKI, 2004. 
que en el ámbito intertropical el viento que se desplaza hacia el sur se desplazará siempre de Este a Oeste, siguiente el movimiento diario del Sol que irá calentando áreas situadas sucesivamente al Oeste. Y lo mismo para los vientos que en el hemisferio sur se desplazan hacia el ecuador. Este principio, indica Kant, «es válido para todos los océanos que se encuentran entre los trópicos: el Atlántico, Etiópico, Pacífico e Índico». Y añade que esta desviación de Este a Oeste es tanto mayor cuanto más grande es la distancia de su recorrido desde los trópicos hacia el ecuador. Precisa el filósofo alemán que estos vientos pueden soplar más allá de los trópicos, pero «no mucho más allá de los treinta grados de latitud», aunque comenta el caso del «viento sur» que sopla en las costas de Chile y Perú y que no es sino el alisio que sopla en esa parte del Pacífico sur americano y que sigue la dirección de la línea de costa.

De manera que en la explicación de los vientos alisios, Kant atribuye su formación a causas exclusivamente térmicas, siguiendo el razonamiento presente en el escritos de Halley (1686), pero no las de origen termo-dinámico de Hadley (1735), aunque estas últimas debía conocerlas, a la vista de la negación del efecto de la rotación en los vientos y el empleo del término «retraso»aparente-de los mismos que se contiene en esta parte de su Geografía Física.

Kant anuncia una cuestión, no conocida en su época pero enormemente significativa. Al referirse al soplo del alisio en la costa de Guinea señala que allí el alisio es del Oeste, en lugar de tener una componente de viento del Este, y explica que ello se debe a que el aire «se calienta más sobre Guinea que sobre el mar». En el fondo está ofreciendo una explicación a la zona de doldrums (calmas) con predominio de vientos de componente occidental existente en las proximidades de la línea ecuatorial.

El mismo origen térmico es el que permite a Kant explicar, como se ha señalado, el funcionamiento de los vientos de mar y tierra (brisas). Aquí se alternan los dos primeros principios generales que Kant había señalado para el soplo del viento. La brisa de mar a tierra tiene que ver con el mayor calentamiento diurno de la superficie terrestre que favorece el movimiento del aire desde las zonas más frías (mar) hacia tierra. Por su parte, la explicación del terral o brisa de tierra se relaciona con el segundo principio general del viento, es decir, aquel según el cual si una zona se enfría poco a poco, el aire situado sobre ella se contrae y deja espacio para que sea ocupado por aire cálido; esto es lo que ocurre por la tarde y noche, según explica Kant -erróneamente-con 
el mar, que se enfría mucho antes que la superficie terrestre obligando a contraerse al aire situado sobre él y, de este modo, permite que el aire de tierra, más caliente, ocupe ese espacio generando así un flujo desde la tierra hacia el mar. Es una explicación errónea del mecanismo de las brisas puesto que no tiene en cuenta el agua del mar que tarda en calentarse y enfriarse bastante más que una superficie continental. De manera que al no incluir la presión atmosférica como elemento clave, en la aparición de los flujos de brisa de mar y tierra, tal y como haría Vilhelm Bjerknes ${ }^{22}$ a comienzos del siglo XX, no encuentra una explicación satisfactoria en el texto de Kant.

Otro apartado importante de la Geografía Física de Kant es el dedicado a la explicación de los vientos monzones o «vientos periódicos» (Figura 3). A través de varias páginas de su obra, el filósofo explica la causa del soplo de estos vientos cambiantes a lo largo del año y que soplan en los mares de todo el cinturón cálido. Pero Kant va más allá e indica que esta explicación sirve de «teoría general de todos los vientos permanente, periódicos y de la mayoría de los cambiantes». De manera que resulta un tanto llamativo que manifieste esta intención casi al final del apartado dedicado a los vientos de su manual, cuando algunos apartados antes, ya había señalado la causa, según él, del soplo de los trade winds. Y resulta, también, contradictorio, puesto que en este nuevo apartado dedicado a los monzones, la explicación causal de los mismos, difiere un tanto de la dada para los vientos alisios, al incorporar, ahora sí, la influencia de la rotación terrestre. En realidad, Kant, nos propone en este epígrafe de su tratado una serie de principios o razonamientos generales sobre el movimiento de los vientos en la superficie terrestre y su relación con la mencionada rotación terrestre, aunque sin abandonar ni mucho menos la causa térmica como origen primero del funcionamiento de la dinámica atmosférica general.

22. El primer teorema de la circulación del aire de Vilhelm Bjerknes señala que «si los gradientes de presión y de temperatura tienen direcciones distintas, se crea energía que fuerza al aire a circular en el sentido que lleva desde el extremo del vector gradiente de presión al del vector gradiente de temperatura, por el camino más corto». La explicación del circuito de brisas (diurna y nocturna) encuentra razonamiento satisfactorio en este principio. Estos principios están recogidos en las obras de V. Bjerknes «Das dynamische Prinzip der Zirkulationsbewegungen in der Atmosphäre». Meteorologiste Zeitschrift. 17 (1900): 97-106, 45-56 y «Zirkulation relativ zu der Erde», Meteorologische Zeitschrift 19 (1902): 97-108. Vid. MEDINA ISABEL, 1976: 13-19. 


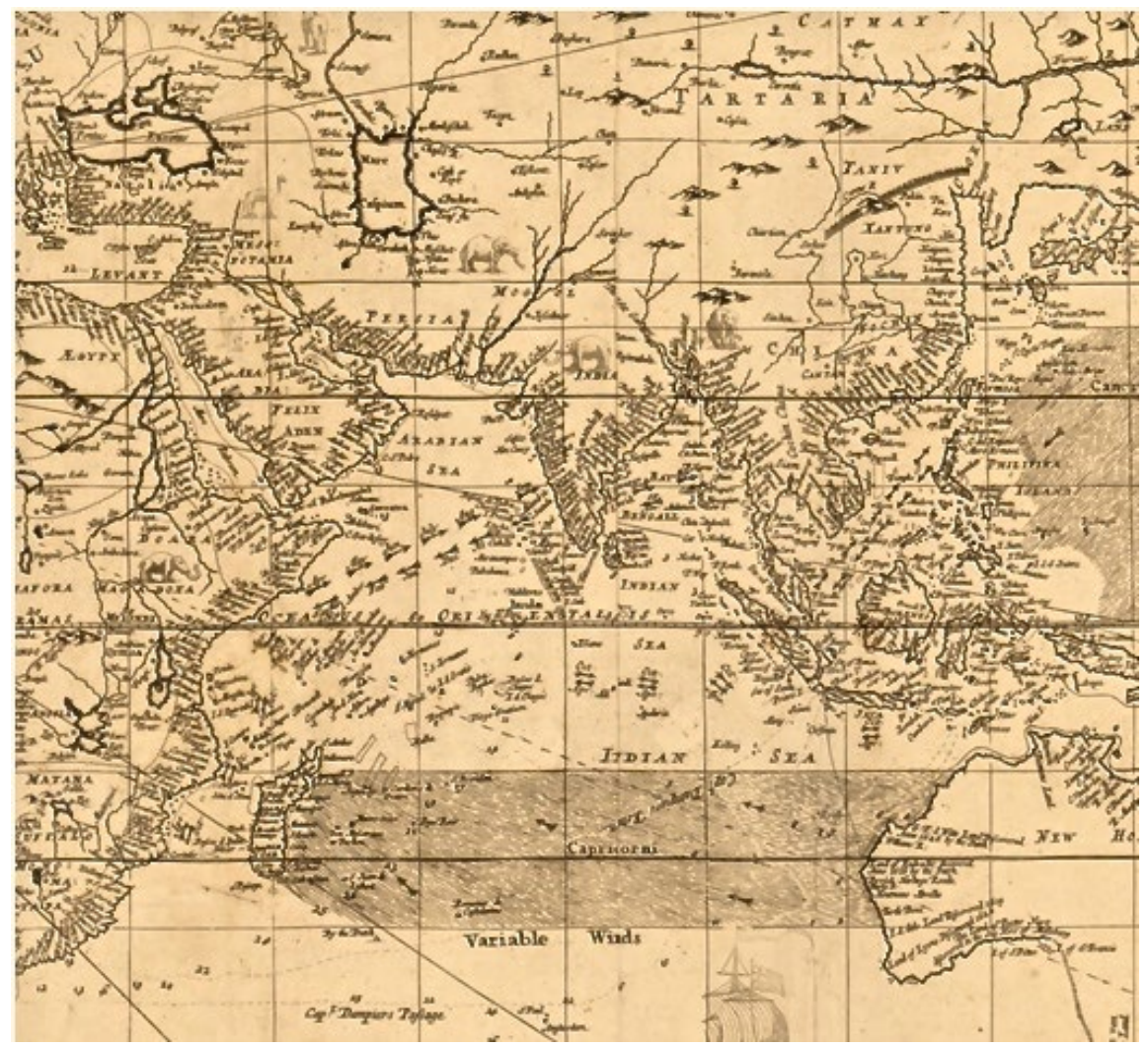

Figura 3.Representación del área afectada por los vientos alisios (sombreado) y por los monzones de invierno y verano (con flechas) en Asia meridional y oriental.

Fuente: Detalle del planisferio «A New and Correct Map of the World, According to Mr. Edward Wright Commonly Called Mercator's Projection with a View of the Winds and Variation», Samuel Thornton, Londres, 1711.

En efecto, Kant, afirma que,

Como la Tierra rota alrededor de su eje, las partes de su superficie describen mayores paralelos, cuanto más cerca se encuentran del ecuador, y más pequeños cuanto más cerca del polo se encuentran, y el aire que cubre la Tierra sufre en todas partes, en ausencia de viento, el mismo movimiento que los elementos de la superficie de la Tierra, sobre los que se mueve. Por lo tanto, el aire del ecuador se mueve del poniente hacia el levante con mayor 
velocidad, que el que se encuentra entre los trópicos y éste más deprisa que el que se haya entre los círculos polares.

Y para explicar la formación de los vientos que se aproximan desde latitudes medias o subtropicales hacia el ecuador, añade,

pero salido de tales zonas de la tierra -latitudes medias o subtropicalesdonde por causa de los pequeños paralelos en los que se encontraba tenía poca velocidad del poniente al levante, como los elementos de la superficie de la Tierra, que se encuentran más próximos al ecuador y hacia los cuales se mueve, así será retrasado, porque no tiene un movimiento tan fuerte de Oeste a Este, lo que unido a la dirección norte produce en nuestro hemisferio un viento noreste, será tanto más viento norte cuando más se aproxime al ecuador, desviado en un viento noreste. Y en el hemisferio sur será un viento sur transformado en uno sudeste por los mismos motivos.

El cuadro adjunto (cuadro 1) resume los tres principios básicos de circulación de vientos sobre la superficie terrestre, según Kant, y el giro que experimentan en relación con la velocidad de rotación de la Tierra en cada ámbito latitudinal.

A estos principios se une la ley general que explicaría el movimiento de los vientos de forma genérica y que se contiene, como se ha indicado, al inicio de la sección «Atmósfera» de la Geografía Física (vid. supra), según la cual el viento sopla siempre de los sectores fríos a los cálidos para compensar la menor densidad y el carácter lábil del aire situado sobre áreas con una mayor temperatura. Con ello y con los tres principios básicos de dinámica atmosférica que ahora añade en su obra, Kant nos propone su explicación de los vientos «monzones» que resultarían de la combinación de los principios 2 y 3. Vuelve, de este modo, el filósofo de Köningsberg a presentar un nuevo intento de explicación de los monzones que realmente sirve como explicación, asimismo, de los vientos alisios, ahora teñida con las ideas sobre los trade winds que Hadley había propuesto en su famoso, pero poco seguido en su época, artículo sobre el mecanismo de los vientos alisios publicado en la revista Philosophical Transactions en 1735.

Realmente la descripción del mecanismo de los monzones ocupa apenas un párrafo, pocas líneas después de la descripción de estos principios o razonamientos sobre el funcionamiento de la dinámica atmosférica. Y en esta descripción del funcionamiento de los vientos monzones, Kant retoma el principio 
Cuadro n. ${ }^{\circ} 1$. Principios de circulación de vientos propuestos por Kant en su Geografía Física

\begin{tabular}{|c|c|c|}
\hline Principio ${ }^{\circ} .^{o} 1$ & Principio $n .^{\circ} 2$ & Principio n. ${ }^{\circ} 3$ \\
\hline $\begin{array}{l}\text { «El viento sopla de W } \\
\text { a E más rápido en el } \\
\text { Ecuador que en latitudes } \\
\text { medias y este último más } \\
\text { rápido que en los círculos } \\
\text { polares». } \\
\text { Este hecho está en } \\
\text { relación con la propia } \\
\text { velocidad de rotación de } \\
\text { la tierra en cada latitud. } \\
\text { Más veloz en el Ecuador y } \\
\text { menos en los Polos }\end{array}$ & $\begin{array}{l}\text { «El viento que se desplaza } \\
\text { del Ecuador hacia el Polo } \\
\text { es primero un viento del } \\
\text { Sur que se transforma } \\
\text { luego en viento del SW } \\
\text { (hemisferio norte) puesto } \\
\text { que en latitudes alejadas } \\
\text { del Ecuador ese viento } \\
\text { sopla con una velocidad } \\
\text { mayor que la existente por } \\
\text { rotación terrestre en dicha } \\
\text { latitud.» } \\
\text { En el hemisferio Sur el } \\
\text { viento que procede del } \\
\text { Ecuador se transformará } \\
\text { en un viento del NW, por } \\
\text { la misma razón. }\end{array}$ & $\begin{array}{l}\text { «El viento del NW que se } \\
\text { desplaza hacia el Ecuador } \\
\text { experimenta un giro y se } \\
\text { transforma en viento del } \\
\text { NE debido a que «queda } \\
\text { retrasado» en latitudes } \\
\text { ecuatoriales respecto a } \\
\text { la propia velocidad de } \\
\text { rotación de la Tierra en } \\
\text { estas latitudes.» } \\
\text { En el hemisferio Sur el } \\
\text { viento del SW que se } \\
\text { desplaza hacia el Ecuador } \\
\text { se transforme en viento del } \\
\text { SE por la misma razón }\end{array}$ \\
\hline$<$ & & ק్ \\
\hline
\end{tabular}

Elaboración propia.

«térmico» como causa exclusiva de los mismos: el mayor calentamiento de la superficie terrestre en el área continental del sur de Asia entre marzo y septiembre origina que el viento del océano Índico se desplace hacia el continente con una trayectoria sudoeste. Esto, añade Kant, «ocurre en el hemisferio sur, solo en los meses restantes de forma que el aire del hemisferio norte será desplazado hasta aquí y producirá un viento noroeste». Así se explica el circuito estacional de los monzones. En los períodos de alternancia entre una circulación (verano) y otra (invierno), en cada hemisferio, «predominarán 
calmas y huracanes». Se trata de una apreciación certera porque, en efecto, los ciclones tropicales en esta parte del mundo -sur de Asia y océano Índicotienen lugar en los equinoccios de primavera y otoño, frente a otras zonas del mundo afectadas por este fenómeno meteorológico - p.e. Caribe- donde se desarrollan solo en un único período anual, entre finales del verano y comienzos del otoño.

La figura adjunta presenta la propuesta de explicación causal de los monzones, en el hemisferio norte, que se recoge en la obra del filósofo alemán (vid. figura 4).

Figura 4. Explicación de los vientos monzones de verano e invierno propuesta por I. Kant en su Geografía Física

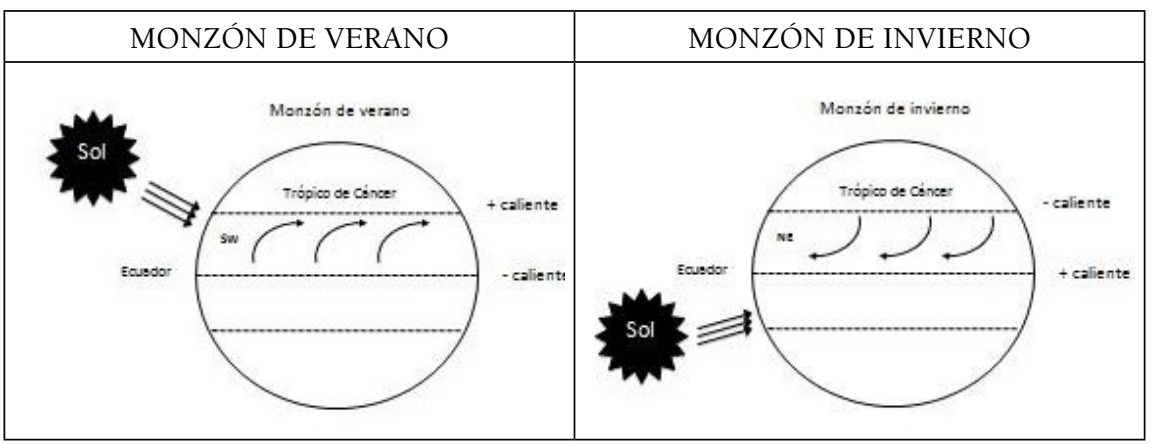

Elaboración propia.

Un último apartado dedicado al viento, dentro de la Geografía Física de Kant, amplia estas ideas sobre el movimiento de los vientos que tan interesantes resultan en la obra del filósofo. En efecto, en el apartado titulado «Algunas otras leyes de la alternancia de los vientos», Kant nos propone una explicación sobre la circulación de los vientos en latitudes medias, que avanza lo que años más tarde, Dove formularía como «ley del giro» (1827). En efecto, afirma Kant que «en nuestro hemisferio norte los vientos acostumbran, cuando se mueven del Norte al Noreste, a finalizar todos los giros de izquierda a derecha, a saber Este, Sur y Oeste», y añade «solo los vientos de un modo opuesto se mueven del Norte al Oeste, no acostumbran jamás a recorrer el círculo 
entero». Y en el hemisferio sur a la inversa dado que «el recorrido del Sol es de derecha a izquierda, como ha constatado Don Ulloa en el Pacífico ${ }^{23}$

Kant hace responsable último de este movimiento al Sol: «el recorrido del Sol, señala, desvía de una manera natural el viento norte en otro noreste; solo si se le resiste el viento del Sur será completamente del Este; entonces empieza el viento del Sur a regresar y por su combinación con el viento del Este se hace sudeste, después completamente del Sur, entonces sudoeste y si existe resistencia del aire del norte completamente del oeste». Años más tarde, Dove incluirá en la explicación del giro del viento a la rotación terrestre, que resulta básica para poder entender el movimiento del aire que se desplaza latitudinalmente ${ }^{24}$.

Hay, por último, una propuesta de «esquema de circulación atmosférica general», aunque Kant no lo propone como tal ni, por supuesto, lo dibuja en su obra ${ }^{25}$. En efecto, afirma Kant que «los vientos son más variables en la mitad entre un polo y el ecuador. Tanto en la zona cálida y regiones adyacentes como en el cinturón frío y zonas vecinas son mucho más constantes». Y termina señalando los cambios en el viento originados en una columna atmosférica, que pueden llegar a provocar «calmas y tormentas repentinamente o viento cambiante en los territorios bajos». Este último es un aspecto de gran interés porque inaugura la serie de estudios sobre el espesor de las capas atmosféricas que tendrán en la navegación aerostática un hito principal, aunque será en el siglo Xx, con el desarrollo de la aviación y, posteriormente, de los satélites de finalidad meteorológica, cuando adquiera su completa y detallada explicación.

23. Se trata de Antonio de Ulloa cuya obra conocía Kant y fue utilizada como bibliografía de consulta en sus apuntes de Geografía Física.

24. Dove no conocerá hasta 1846 la explicación del giro de los vientos incluida en la Geografía Física de Kant, por lo que no pudo tenerla en cuenta en la formulación inicial de su «ley del Giro» en 1827. Carramolino, 3 (1994): 95-119.

25. Uno de los problemas de la Geografía Física de Kant es que no se incluye ninguna ilustración o dibujo de sus propuestas de explicación de los fenómenos atmosféricos, aunque no parece extraño que los utilizara en sus clases. 


\section{Epílogo}

Las ciencias atmosféricas (meteorología y climatología) experimentarán un empuje definitivo a lo largo del siglo XIX y su consolidación definitiva a partir de las primeras décadas del siglo Xx con la aparición de las ideas contemporáneas sobre circulación atmosférica (teoría frontogenética, corrientes en chorro). Pero todo ello no hubiera sido posible sin el desarrollo de las propuestas sobre circulación del aire y, especialmente, de los vientos que soplan en el ámbito intertropical (alisios y monzones). Las ideas de Bacon, Halley, Hadley, Varenio y Kant sobre la dinámica atmosférica en el ámbito intertropical, espacio geográfico de especial importancia en la época por sus implicaciones para el comercio marítimo, resultan básicas para el posterior avance de la meteorología y climatología. La superación de las ideas aristotélicas sobre el soplo del viento, que ya se había apuntado en autores como José de Acosta o el propio Galileo, se consolidará en los siglos XVII y XVIII con la inclusión del efecto de la velocidad de rotación terrestre sobre el desplazamiento del aire que pierde o gana latitud geográfica. No obstante, no será hasta la incorporación de las diferencias de presión atmosférica como elemento, asimismo, determinante en el movimiento del aire, ya en el siglo $\mathrm{XX}$, cuando se desentrañe la causa última de la circulación del viento y el soplo del mismo en diferentes zonas terrestres. La disciplina climática cuenta con tres figuras principales en la Edad Moderna: Acosta, Varenio y Kant. En sus escritos, que se suceden en el tiempo, se recoge lo esencial del avance en el conocimiento de la atmósfera terrestre en esos siglos; se superan las ideas clásicas de Aristóteles y Plinio el Viejo y se prefigura la explicación de los movimientos superficiales del aire de los siglos posteriores. En este proceso, la explicación del mecanismo de los vientos que circulan en los trópicos servirá de principal acicate para consolidar a la climatología como disciplina científica dentro de las ciencias de la naturaleza.

\section{Bibliografía}

Alberola RomÁ, Armando, Los cambios climáticos. La Pequeña Edad del Hielo en España, Madrid, Ed. Cátedra, 2014.

ArCIMIS, Augusto, Meteorología, Barcelona, Manuales Soler n. ${ }^{\circ}$ XVIII, 1902. ARnaU, Juan, Manual de filosofía portátil, Madrid. Ediciones Atalanta, 2014. 
BACON, Francis, History of the winds, Londres, Matthew Lownes \& William Barret, 1622. Disponible en: http://www.sirbacon.org/naturalhistorywinds. htm

BARry, Roger G. y ChORLey, Richard J., Atmosphere, Weather and Climate, (7.a ed.), London/New York, Ed. Routledge, 1999.

Belaval, Yvon (dir.), La filosofía alemana, de Leibniz a Hegel. Historia de la Filosofía, México, Ed. Siglo XXI, 1982.

BOROWSKI, Ludwin Ernest, Relato de la vida y el carácter de Immanuel Kant, Madrid, Tecnos, 1993.

BRAzDIL, Rudolf et al., «Historical climatology in Europe. The state of the art», Climate Change, 70 (2005): 363-430.

BRITO, Arlete de Jesus y SCHUBRING, Gert, «La práctica de la matemática «normal» en el siglo XVII: el caso de la Geografía Matemática de Varenius», Llull, 31/67 (2008): 41-60. Disponible en: https://dialnet.unirioja.es/ejemplar/221450

Broc, Numa, La Géographie de la Renaissance (1420-1620), Paris, Bibliothèque Nationale, 1980.

CAPEL, Horacio, «La personalidad geográfica de Varenio», en Bernhard Varenio y Horacio Capel (ed. y estud. prel.), Geografía General en la que se explican las propiedades generales de la Tierra, Barcelona, Ediciones de la Universidad de Barcelona, 1980: 11-84. Disponible en: http://hdl.handle.net/2445/67064 CAPEL, Horacio, Filosofía y Ciencia en la Geografía contemporánea, Barcelona, Ediciones del Serbal, 2012.

Carramolino, David, «La «Ley de Giro» (Drehungsgesetz) de Dove (1827) y el nacimiento de la dinámica atmosférica en Alemania», Endoxa: Series Filosóficas, 3 (1994): 95-119. Disponible en: http://revistas.uned.es/index. php/endoxa/article/viewFile/4802/4622

ChAmbers, Ephraim, Voz «Trade-winds», en Cyclopaedia, or an Universal Dictionary of Arts and Sciences, Londres, J. and J. Knapton et al., 1728. Disponible en: www.cyclopaedia.org [consultado el 10 de noviembre de 2016] Cohen-Halimi, Michèle, «Le Géographe de Königsberg», en Immanuel Kant, Michèle Cohen-Halimi y Max Marcuzzi (trads.), Géographie. Physische Geographie, Paris, Aubier, 1999: 9-40.

COPLESTON, Frederick, Historia de la Filosofía. Vol. 3: De la Filosofía Kantiana al Idealismo, Barcelona, Ed. Ariel, 2011.

DesCARTES, René, Discurso del método. Madrid. Ed. Akal, 2007. 
ELDEN, Stuart, «Reassessing Kant's geography», Journal of Historical Geography, 35/1 (2009): 3-25. http://dx.doi.org/10.1016/j.jhg.2008.06.001

ELDEN, Stuart, «Reintroducing Kant's geography», en Stuart Elden y Eduardo Mendieta (eds.), Reading Kant's Geography, Albany, State University of New York Press, 2011: 1-15.

ELDEN, Stuart, «Leibniz and geography: geologist, paleontologist, biologist, historian, political theorist and geopolitician», Geographica Helvetica, 68 (2013): 81-93. http://dx.doi.org/10.5194/gh-68-81-2013

FATONE, Vicente, Kant, profesor de geografía, Buenos Aires, Biblioteca Virtual Universal, 2003, 5 p. Disponible en http://www.biblioteca.org.ar/libros/1272. pdf [consultado el 15 de noviembre de 2016]

Flammarion, Camille, La atmósfera: descripción de los grandes fenómenos de la naturaleza, Madrid, Imprenta y Librería de Gaspar Editores, 1875. Disponible en https://books.google.es

Gil OlCinA, Antonio y OlCina CANTOS, Jorge, Climatología general, Barcelona, Ed. Ariel, 1997.

Gil Olcina, Antonio y Olcina Cantos, Jorge. Tratado de Climatología, Alicante, Publicaciones de la Universitat d'Alacant, 2017.

GlaCKEn, Clarence J., Huellas en la playa de Rodas, Barcelona, Ed. del Serbal, 1996.

Grapotte, Sofie, Lequan, Mai y Ruffing, Margit, (eds.), Kant et les sciences: Un dialogue philosophique avec la pluralité des savoirs, Paris, Librairie Philosophique J. Vrin, 2011.

HADLEY, George, "Concerning the cause of the general trade-winds», Philosophical Transactions, 39/436-444 (1735): 58-62. http://dx.doi.org/10.1098/ rstl.1735.0014

Halley, Edmund, «An Historical Account of the Trade Winds, and Monsoons, observable in the Seas between and near the Tropicks, with an attempt to assign the phisical cause of the said winds», Philosophical Transactions, 16/179-191 (1686): 153-168. http://dx.doi.org/10.1098/rstl.1686.0026

HARSTSHORNE, Richard (1991) «El concepto de geografía como ciencia del espacio: de Kant y Humboldt a Hettner», Documents d'Anàlisi Geogràfica, 18 (1991): 31-54. Disponible en: http://ddd.uab.cat/record/16312

Humboldt, Alexander von, Cosmos. Ensayo de una descripción física del mundo. Buenos Aires. Ed. Glem, 1944. 
JuAn, Jorge, Compendio de navegación para el uso de los cavalleros Guardias Marinas, Cádiz, en la Academia de los mismos cavalleros, 1757. Disponible en el Portal dedicado a Jorge Juan Santacilia (Armando Alberola Romá y Rosario Die Maculet, eds.): http://www.cervantesvirtual.com/portales/jorge_juan_santacilia/obra/compendio-de-navegacion-para-el-uso-de-los-cavalleros-guardias-marinas--0/ [consultado el 2 de diciembre de 2016]

JuAn, Jorge y Ulloa, Antonio de, Relación histórica del viage a la América meridional hecho de orden de Su Magestad para medir algunos grados de Meridiano terrestre, y venir por ellos en conocimiento de la verdadera figura y magnitud de la Tierra, con otras varias observaciones astronómicas, y phisicas, Madrid, Antonio Marín, 1748. Disponible en el Portal dedicado a Jorge Juan Santacilia (Armando Alberola Romá y Rosario Die Maculet, eds.): http://www.cervantesvirtual.com/portales/jorge_juan_santacilia/obra/relacion-historica-del-viagea-la-america-meridional-hecho-de-orden-de-s-mag-para-medir-algunos-grados-de-meridiano-terrestre-y-venir-por-ellos-en-conocimiento-de-la-verdadera-figura-y-magnitud-de-la-tierra-con-otras-observaciones-astronomicas-y-phisicas/

JuAN, Jorge y Ulloa, Antonio de, Observaciones astronomicas y phisicas hechas de orden de S. Mag. en los Reynos de Perú... Madrid, Imprenta Real de la Gazeta, 1773. Disponible en el Portal dedicado a Jorge Juan Santacilia (Armando Alberola Romá y Rosario Die Maculet, eds.): http://www.cervantesvirtual. com/portales/jorge_juan_santacilia/obra/observaciones-astronomicas-yphisicas-hechas-de-orden-de-s-m-en-los-reynos-del-peru--0/ [consultado el 2 de diciembre de 2016]

KANT, Inmanuel, Annonce du programme des leçons de M. E. Kant durant le semestre d'hiver 1765-1766, Paris, Librairie Philosophique J. Vrin, 1966.

KANT, Inmanuel, Filosofía de la Historia, México, Fondo de Cultura Económica, 1981.

Kerszberg, Pierre, «La création en mouvement. Essai sur le sens philosophique d'une interrogation cosmologique fondamentale dans la Théorie du Ciel», en Immanuel Kant, Pierre Kerszberg, Anne-Marie Roviello y Jean Seidengart (trad., intr. y not.), Histoire Générale de la Nature et Théorie du Ciel, Paris, Librairie Philosophique J. Vrin, 1984: 205-259.

LORENTE, José María, Meteorología. Barcelona, Editorial Labor, 1930. 
MANZO, Silvia, «Francis Bacon y la concepción aristotélica del movimiento en los siglos XVI y XVII», Revista de Filosofía, 29/1 (2004): 77-97. Disponible en: http://revistas.ucm.es/index.php/RESF/article/view/RESF0404120077A

Marcuzzi, Max, «La Géographie kantienne: délimitation de la discipline», en Immanuel Kant, Michèle Cohen-Halimi y Max Marcuzzi (trads.), Géographie. Physische Geographie, Paris, Aubier, 1999: 40-55.

MarTín Vide, Javier, Fundamentos de Climatología Analítica. Madrid, Ed. Síntesis, 1991.

Martonne, Emmanuel de, Tratado de Geografía Física. Tomo I. Nociones Generales, Clima, Hidrología, (ed. en español), Barcelona, Ed. Juventud, 1964.

MAS Galvañ, Cayetano, «El océano vertical: La cuestión de las altitudes en Jorge Juan y Antonio de Ulloa», en Armando Alberola Romá, Cayetano Mas Galvañ y Rosario Die Maculet (eds.), Jorge Juan Santacilia en la España de la Ilustración, San Vicente del Raspeig, Servicio de Publicaciones de la Universidad de Alicante, 2015: 63-86.

Medina Isabel, Mariano, Meteorología básica sinóptica. Madrid, Ed. Paraninfo, 1976.

OlCina Cantos, Jorge, «Clasificación de las nubes: de Lamarck y Howard al Atlas Internacional de Nubes», en Armando Alberola Romá (coord.), Clima, Naturaleza y desastre. España e Hispanoamérica durante la Edad Moderna, Valencia, Publicacions de la Universitat de València, 2013: 193-224.

OlCina CANTOS, Jorge, «Referencias atmosféricas y avances para la ciencia climática en la obra de José de Acosta» Scripta Nova. Revista Electrónica de Geografía y Ciencias Sociales, XVIII/478 (2014a). Disponible en: http://www. ub.es/geocrit/sn/sn-478.htm

OlCina CANTOS, Jorge, «Enseñanzas climáticas en la obra de Kant», Anales de Geografía de la Universidad Complutense, 34/2(2014b): 119-162. http:// revistas.ucm.es/index.php/AGUC/article/view/47075

OlCINA CANTOS, Jorge, «La ciencia climática en el siglo XVII: la aportación de Varenio en la Geografía General», en José Fernando Vera Rebollo, Jorge Olcina Cantos y María Hernández Hernández (eds.), Paisaje, cultura territorial y vivencia de la Geografía. Libro homenaje al profesor Alfredo Morales Gil, San Vicente del Raspeig, Servicio de Publicaciones de la Universidad de Alicante, 2016: 815-852.

ORTEGa VAlCÁRCEL, José, Los horizontes de la geografía. Teoría de la geografía. Barcelona, Ed. Ariel, 2000. 
Ortega y Gasset, José, Kant, Hegel, Scheler. Madrid, Revista de Occidente en Alianza Editorial, 1983.

PelKOWSKI, Joaquín, «La anemología kantiana: contexto histórico y versión castellana», Revista del Aficionado a la Meteorología (RAM), 2004. Disponible en: http://www.tiempo.com/ram/33112/la-anemologia-kantiana-contextohistorico-y-version-castellana/. [consultado el 15 de noviembre de 2016]

PelkowsKi, Joaquín, «El certamen de 1746 acerca de la causa de los vientos generales», Meteorología Colombiana, 9 (2005): 93-104. Disponible en: http:// www.divulgameteo.es/uploads/Certamen-1746.pdf

Pelkowski, Joaquín, «Teoría de los Alisios durante la Ilustración: Primera parte», Boletín de la Asociación Meteorológica Española, 5/14 (2006): 24-30. Disponible en: http://pkp.ame-web.org/index.php/TyC/article/view/494

PelKowSKI, Joaquín, «Teoría de los Alisios durante la Ilustración: Segunda parte», Boletín de la Asociación Meteorológica Española, 5/15 (2007): 20-27. Disponible en: http://pkp.ame-web.org/index.php/TyC/article/view/483

PERSSON, Anders O., «Hadley's Principle: Understanding and Misunderstanding the Trade Winds», History of Meteorology, 3 (2006): 17-42. Disponible en: http://www.meteohistory.org/2006historyofmeteorology3/2persson_hadley. pdf

REBOK, Sandra, «The influence of Bernhard Varenius in the geographical works of Thomas Jefferson and Alexander von Humboldt», en Margret Schuchard (ed.), Bernhard Varenius (1622-1650), Leiden/Boston, Ed. Brill, 2007: 259270. https://doi.org/10.1163/ej.9789004163638.i-351.89

REDONDO ÁlVARO, Francisco Luis, «Algunos rasgos de la revolución científica en el siglo XVII», Boletín del Instituto de Estudios Giennenses, 198 (2008): 565-599. Disponible en: https://dialnet.unirioja.es/ejemplar/224186

Refolio Refolio, M. ${ }^{a}$ del Carmen y López Sancho, José M. ${ }^{a}$, «Un mapa cultural para situar la enseñanza de la ciencia: la revolución científica y el fin del aristotelismo», El CSIC en la Escuela. Investigación sobre la enseñanza de la ciencia en el aula, 1(2011): 81-92. Disponible en: http://digital.csic.es/ handle/10261/83293. [consultado el 10 de noviembre de 2016]

REguera RodrígueZ, Antonio T., «Newton y Feijoo. Un episodio en la historia de la difusión de las ideas científicas (I y II) », Contextos XIX-XX/37-40 (20012002): 283-344. Disponible en: https://buleria.unileon.es/handle/10612/405. RIBAS, Alexandre Domingues, Cosmologia e Geografia Física em Immanuel Kant. Tesis Doctoral. Campinas, Universidade Estadual de Campinas, 2011. (Disponible 
en: http://www.bibliotecadigital.unicamp.br/document/?code=000804783) [consultado el 21 de noviembre de 2016]

RiBAS, Alexandre Domingues y VitTE, Antonio Carlos, "O Curso de Geografía Física de Immanuel Kant (1724-1804): entre a Cosmología e a Estética», Biblio 3W. Revista Bibliográfica de Geografía y Ciencias Sociales, XIV/844 (2009). Disponible en: http://www.ub.es/geocrit/b3w-844.htm [consultado el 15 de noviembre de 2016]

ROJAS SALAZAR, Temístocles, «Los aportes de Kant a la geografía», Terra Nueva Etapa, XXIII/34 (2007): 11-33. Disponible en: http://www.redalyc.org/ articulo.oa?id=72103402

RUSSELl, Bertrand, Historia de la Filosofía Occidental. Tomo II: La filosofía moderna, Madrid, Ed. Austral, 2010.

SCHUCHARD, Margret, "Notes on Geographia Generalis and its introduction to England and North America», en Margret Schuchard (ed.), Bernhard Varenius (1622-1650), Leiden/Boston, Ed. Brill, 2007: 227-238. https://doi. org/10.1163/ej.9789004163638.i-351.76

SEIDENGART, Jean, «Genèse et structure de la cosmologie kantienne précritique», en Immanuel Kant, Pierre Kerszberg, Anne-Marie Roviello y Jean Seidengart (trad., intr. y not.), Histoire Générale de la Nature et Théorie du Ciel, Paris, Librairie Philosophique J. Vrin, 1984: 7-59.

SUTTON, Graham, "Theories of the circulation of the Earth's atmosphere», The Observatory, 80/918 (1960): 169-190. Disponible en: http://adsabs.harvard. edu/full/1960Obs....80..169S

UlloA, Antonio de, Noticias Americanas. Madrid, Imprenta de Don Francisco Manuel de Mena, 1792. Disponible en el Portal dedicado a Jorge Juan Santacilia (Armando Alberola Romá y Rosario Die Maculet, eds.): http:// www.cervantesvirtual.com/obra/noticias-americanas--1/ [consultado el 2 de diciembre de 2016]

Uriarte Cantolla, Antón, Historia del Clima de la Tierra. Vitoria, Servicio Central de Publicaciones del Gobierno Vasco, 2002.

URTEAGA, Luis, "La teoría de los climas y los orígenes del ambientalismo», Geocrítica, XVIII/99 (1993). Disponible en http://www.ub.edu/geocrit/geo99. htm. [consultado el 22 de noviembre de 2016]

VARENIO, Bernhardus, Geographia generalis, in qua affectiones generales telluris explicantur. Amstelodami, Ex Officina Elzeviriana, 1664. Disponible en https://books.google.com 
VARENIO, Bernhard y CAPEL, Horacio (ed. y estud. prel.), Geografía General en la que se explican las propiedades generales de la Tierra, Barcelona, Ediciones de la Universidad de Barcelona, 1974.

VERMIJ, Rienk (2007) «Varenius and the world of learning in the Dutch Republic», en Margret Schuchard (ed.), Bernhard Varenius (1622-1650), Leiden/Boston, Ed. Brill, 2007: 99-116. https://doi.org/10.1163/ej.9789004163638.i-351.30 VicuÑA, Gumersindo, Manual de meteorología popular, Madrid, Tip. de G. Estrada, 1880. Disponible en: http://bdh-rd.bne.es/viewer.vm?id=0000058919\&page=1 WARNTZ, William, «Newton, the Newtonians, and the Geographia Generalis Varenii», Annals of the Asociation of American Geographers, 79/2 (1989): 165191. Disponible en: http://www.jstor.org/stable/2563251.

WatKInS, Eric (ed.), Kant and the Sciences, Oxford, Oxford University Press, 2001.

Watkins, Eric (ed.), Natural Science. The Cambridge edition of the Works of Immanuel Kant in translation, San Diego, University of California, 2012.

WeINBERG, Steven, Explicar el mundo. El descubrimiento de la ciencia moderna. Madrid, Ed. Taurus, 2015.

Welti, Reinaldo, La explicación del cielo azul. Una aventura del pensamiento, Rosario, Vesperi Refulgens Editora, 2013. Disponible en: https://tecnoedu. com/Download/LaExplicacionDelAzulDelCieloWelti.pdf 